\title{
Erratum to: Self-similar solution of cylindrical shock wave propagation in a rotational axisymmetric mixture of a non-ideal gas and small solid particles
}

\author{
G. Nath
}

Published online: 26 July 2012

(C) Springer Science+Business Media B.V. 2012

\section{Erratum to: Meccanica}

DOI 10.1007/s11012-012-9543-4

The authors wish to apologize for any inconvenience caused due to the fact that erroneous versions of several equations were published. Please find below the correct equations mentioned that should be regarded by the reader as the final versions

(1) Below Eq. (34)

$$
a_{a}=\left(\frac{\left[\left(1-Z_{a}\right)\left\{\Gamma\left(1-Z_{a}\right)-2 Z_{a}\right\}+b \rho_{a}\left(1-K_{p}\right)\left\{2 \Gamma\left(1-Z_{a}\right)-Z_{a}\right\}\right] p_{a}}{\rho_{a}\left(1-Z_{a}\right)\left[\left(1-Z_{a}\right)+b \rho_{a}\left(1-K_{p}\right)\right]}\right)^{\frac{1}{2}},
$$

read as

$$
a_{a}=\left(\frac{\left[\Gamma\left(1-Z_{a}\right)^{2}+b \rho_{a}\left(1-K_{p}\right)\left\{2 \Gamma\left(1-Z_{a}\right)+Z_{a}\right\}\right] p_{a}}{\rho_{a}\left(1-Z_{a}\right)\left[\left(1-Z_{a}\right)+b \rho_{a}\left(1-K_{p}\right)\right]}\right)^{\frac{1}{2}} .
$$

(2) Below Eq. (34)

$$
M_{e}^{2}=\frac{\left.\left(1-Z_{a}\right)\left[\left(1-Z_{a}\right)+b \rho_{a}\left(1-K_{p}\right)\right]\right] \gamma M^{2}}{\left[\left(1-Z_{a}\right)\left\{\Gamma\left(1-Z_{a}\right)-2 Z_{a}\right\}+b \rho_{a}\left(1-K_{p}\right)\left\{2 \Gamma\left(1-Z_{a}\right)-Z_{a}\right\}\right]},
$$

read as

$$
M_{e}^{2}=\frac{\left(1-Z_{a}\right)\left[\left(1-Z_{a}\right)+b \rho_{a}\left(1-K_{p}\right)\right] \gamma M^{2}}{\left[\Gamma\left(1-Z_{a}\right)^{2}+b \rho_{a}\left(1-K_{p}\right)\left\{2 \Gamma\left(1-Z_{a}\right)+Z_{a}\right\}\right]} .
$$

The online version of the original article can be found under doi:10.1007/s11012-012-9543-4.

G. Nath $(\bowtie)$

Department of Mathematics, National Institute of Technology Raipur, G.E. Road, Raipur 492010, India

e-mail: gn_chaurasia_univgkp@yahoo.in 
(3) Below Eq. (34)

$$
M_{e}^{2}=\frac{W_{s}^{2}}{a_{a}^{2}}=\frac{\left(1-Z_{a}\right) \rho_{a} W_{s}^{2}\left[\left(1-Z_{a}\right)+b \rho_{a}\left(1-K_{p}\right)\right]}{\left[\left(1-Z_{a}\right)\left\{\Gamma\left(1-Z_{a}\right)-2 Z_{a}\right\}+b \rho_{a}\left(1-K_{p}\right)\left\{2 \Gamma\left(1-Z_{a}\right)-Z_{a}\right\}\right] p_{a}},
$$

read as

$$
M_{e}^{2}=\frac{W_{s}^{2}}{a_{a}^{2}}=\frac{\left(1-Z_{a}\right) \rho_{a} W_{s}^{2}\left[\left(1-Z_{a}\right)+b \rho_{a}\left(1-K_{p}\right)\right]}{\left[\Gamma\left(1-Z_{a}\right)^{2}+b \rho_{a}\left(1-K_{p}\right)\left\{2 \Gamma\left(1-Z_{a}\right)+Z_{a}\right\}\right] p_{a}} .
$$

(4) Equation (45)

$$
\begin{aligned}
M_{e}^{2} & =\frac{\left(1-Z_{a}\right)\left[\left(1-Z_{a}\right)+\bar{b}\left(1-K_{p}\right)\right] 2 n(n+1)^{\frac{(n-1)}{(n+1)}}}{\left[\left(1-Z_{a}\right)\left\{\Gamma\left(1-Z_{a}\right)-2 Z_{a}\right\}+\bar{b}\left(1-K_{p}\right)\left\{2 \Gamma\left(1-Z_{a}\right)-Z_{a}\right\}\right] B^{2}}\left(C V_{0}\right)^{\frac{2}{(n+1)}} \\
& =\text { constant, }
\end{aligned}
$$

read as

$$
\begin{aligned}
M_{e}^{2} & =\frac{\left(1-Z_{a}\right)\left[\left(1-Z_{a}\right)+\bar{b}\left(1-K_{p}\right)\right] 2 n(n+1)^{\frac{(n-1)}{(n+1)}}}{\left[\Gamma\left(1-Z_{a}\right)^{2}+\bar{b}\left(1-K_{p}\right)\left\{2 \Gamma\left(1-Z_{a}\right)+Z_{a}\right\}\right] B^{2}}\left(C V_{0}\right)^{\frac{2}{(n+1)}} \\
& =\text { constant. }
\end{aligned}
$$

(5) Equation (63)

$$
a_{m}=\left(\frac{\partial p}{\partial \rho}\right)_{S}^{\frac{1}{2}}=\left[\frac{\left.[(1-Z)\{\Gamma(1-Z)-2 Z)\}+\{2 \Gamma(1-Z)-Z)\} b \rho\left(1-K_{p}\right)\right] p}{(1-Z) \rho\left\{(1-Z)+b \rho\left(1-K_{p}\right)\right\}}\right]^{\frac{1}{2}},
$$

read as

$$
a_{m}=\left(\frac{\partial p}{\partial \rho}\right)_{S}^{\frac{1}{2}}=\left[\frac{\left.\left[\Gamma(1-Z)^{2}+\{2 \Gamma(1-Z)+Z)\right\} b \rho\left(1-K_{p}\right)\right] p}{(1-Z) \rho\left\{(1-Z)+b \rho\left(1-K_{p}\right)\right\}}\right]^{\frac{1}{2}} .
$$

(6) Equation (64)

$$
\begin{aligned}
C_{a d i} & =-\rho\left(\frac{\partial}{\partial p}\left(\frac{1}{\rho}\right)\right)_{S} \\
& =\frac{1}{\rho a_{m}^{2}}=\frac{(1-Z)\left[(1-Z)+b \rho\left(1-K_{p}\right)\right]}{\left[(1-Z)\{\Gamma((1-Z)-2 Z)\}+\{2 \Gamma(1-Z)-Z\} b \rho\left(1-K_{p}\right)\right] p},
\end{aligned}
$$

read as

$$
\begin{aligned}
C_{a d i} & =-\rho\left(\frac{\partial}{\partial p}\left(\frac{1}{\rho}\right)\right)_{S} \\
& =\frac{1}{\rho a_{m}^{2}}=\frac{(1-Z)\left[(1-Z)+b \rho\left(1-K_{p}\right)\right]}{\left[\Gamma(1-Z)^{2}+\{2 \Gamma(1-Z)+Z\} b \rho\left(1-K_{p}\right)\right] p} .
\end{aligned}
$$

(7) Below Eq. (69)

$$
J=J(\eta)=\frac{P Z_{a}^{2} D^{2}\left[1-\bar{b}\left(1-K_{p}\right)\right]-P-P(\Gamma-1)\left[\left(1-Z_{a} D\right)+\bar{b} D\left(1-K_{p}\right)\right]^{2}}{D\left(1-Z_{a} D\right)\left[\left(1-Z_{a} D\right)+\bar{b} D\left(1-K_{p}\right)\right]},
$$

read as

$$
J=J(\eta)=\frac{P\left[D \bar{b}\left(1-K_{p}\right)\left(Z_{a} D-2\right)-\left(1-Z_{a} D\right)^{2}\right]-P(\Gamma-1)\left[\left(1-Z_{a} D\right)+\bar{b} D\left(1-K_{p}\right)\right]^{2}}{D\left(1-Z_{a} D\right)\left[\left(1-Z_{a} D\right)+\bar{b} D\left(1-K_{p}\right)\right]} .
$$


(8) Equation (75)

$$
\left(C_{a d i}\right) p_{a}=\frac{(n+1)^{2}\left(1-Z_{a} D\right)\left[\left(1-Z_{a} D\right)+\bar{b} D\left(1-K_{p}\right)\right]}{\eta^{2}\left[\left(1-Z_{a} D\right)\left\{\Gamma\left(1-Z_{a} D\right)-2 Z_{a} D\right\}+\bar{b} D\left(1-K_{p}\right)\left\{2 \Gamma\left(1-Z_{a} D\right)-Z_{a} D\right\}\right] \gamma M^{2} P},
$$

read as

$$
\left(C_{a d i}\right) p_{a}=\frac{(n+1)^{2}\left(1-Z_{a} D\right)\left[\left(1-Z_{a} D\right)+\bar{b} D\left(1-K_{p}\right)\right]}{\eta^{2}\left[\Gamma\left(1-Z_{a} D\right)^{2}+\bar{b} D\left(1-K_{p}\right)\left\{2 \Gamma\left(1-Z_{a} D\right)+Z_{a} D\right\}\right] \gamma M^{2} P} .
$$

(9) Equation (77)

$$
\frac{D^{\Gamma+\left(\frac{n}{n+1}\right)}\left[\left(1-Z_{a} D\right)+\bar{b} D\left(1-K_{p}\right)\right]^{\left\{1-\frac{\bar{b}\left(1-K_{p}\right) Z_{a}\left(Z_{a}+1\right)-2 Z_{a}^{2}}{\left.\bar{b}\left(1-K_{p}\right) \bar{b}\left(1-K_{p}\right)-Z_{a}\right]}\right\}}}{\left[1-Z_{a} D\right]^{\left\{Z_{a}+\frac{(\Gamma-1) \bar{b}\left(1-K_{p}\right)}{Z_{a}}\right\}}}=\frac{P \eta^{\left(\frac{2}{n+1}\right)}}{C_{1}}[U-n-1]^{-\left(\frac{n}{n+1}\right)},
$$

read as

$$
\frac{D^{\Gamma+\left(\frac{n}{n+1}\right)}\left[\left(1-Z_{a} D\right)+\bar{b} D\left(1-K_{p}\right)\right]}{\left[1-Z_{a} D\right]^{\left\{1+\frac{(\Gamma-1) \bar{b}(1-K p)}{Z_{a}}\right\}}}=\frac{P \eta^{\left(\frac{2}{n+1}\right)}}{C_{1}}[U-n-1]^{-\left(\frac{n}{n+1}\right)} .
$$

